\title{
Isolation of poliovirus shedding following vaccination in children with antibody deficiency disorders
}

\author{
Nermeen M. Galal ${ }^{1}$, Laila Bassiouny ${ }^{2}$, Eman Nasr² ${ }^{2}$ Naglaa Abdelmeguid ${ }^{2}$ \\ ${ }^{1}$ Cairo University Specialized Paediatric Hospital, Cairo University, Egypt \\ ${ }^{2}$ Regional Laboratory for Enteroviruses, World Health Organization, The Holding Company for Biological Products \\ and Vaccines (VACSERA), Cairo, Egypt
}

\begin{abstract}
Introduction: Prolonged excretion of oral poliovirus may occur in primary antibody deficiency states. Those patients who persistently excrete the virus may pose the risk of aiding viral propagation in the environment. This study therefore aimed to identify the potential for prolonged poliovirus shedding by patients diagnosed with congenital antibody deficiency disorders.

Methodology: A cohort of children later diagnosed with antibody deficiency disorders was included in the study. Patient history was taken for each participant, with emphasis on vaccination data. Laboratory investigations included immunoglobulin profiles and stool sample collection at one month intervals from each patient, with follow-up for six months. The virus isolates were detected using enzyme-linked immunosorbent assay (ELISA) and molecular reverse transcription polymerase chain reaction (RT-PCR) techniques.

Results: On the initial sample screens, one patient revealed excretion one for Sabin-like strain 1 (SL1) and one patient revealed excretion for Sabin like strain 2 (SL2). Only one patient continued to shed the virus (SL1) on three successive samples and on follow-up. There was no correlation between the level of immunoglobulins and duration of virus shedding.

Conclusion: The study demonstrates the low occurrence of prolonged vaccine polioviruses shedding in a group of children exposed to a live vaccine.
\end{abstract}

Key words: primary immunodeficiency; poliomyelitis; prolonged shedding

J Infect Dev Ctries 2012; 6(12):881-885.

(Received 26 October 2011 - Accepted18 January 2012)

Copyright (C) 2012 Galal et al. This is an open-access article distributed under the Creative Commons Attribution License, which permits unrestricted use, distribution, and reproduction in any medium, provided the original work is properly cited.

\section{Introduction}

The global polio eradication program recommends the use of oral poliovirus vaccine (OPV) through National Immunization Days (NID). However, use of OPV is associated with some rare adverse events, including the appearance of cases of vaccineassociated paralytic poliomyelitis (VAPP) among OPV recipients and close contacts, and the occurrence of polio outbreaks associated with circulating vaccine derived poliovirus (cVDPV).

In immunocompetent individuals, the risk of VAPP is very low compared with that of immunodeficient patients [1]. Susceptible individuals usually excrete polioviruses for two to six weeks and occasionally for up to 137 days after they have been immunized with oral poliovirus vaccine [2]. The shedded viruses frequently show increased neurovirulence and are often transmitted to close contacts.

Although immunodeficiencies are listed as a contraindication for receiving OPV, patients with these clinical conditions may occasionally receive the poliovirus live vaccine before their immunodeficiency is diagnosed and/or may be infected with OPV strains excreted by a vaccinee or that is present in the community.

Identification of highly evolved OPV derived strains is an indication of prolonged replication since the administration of the original OPV dose. Such replication could occur in immune deficient individuals, as has been documented for OPV-derived viruses of all three serotypes [3-7]. Persistent poliovirus excretors could be a source of transmission or reintroduction of neurovirulent polioviruses from individuals with immunodeficiency disorders [8].

Sabin viruses can replicate for very long periods in immunodeficient patients (iVDPV), and in some cases, this leads to fatal disease. Recent genetic analysis of virus isolates from an asymptomatic immunodeficient person suggests that infections can continue for as long as 10 or more years after vaccination of immunocompromised persons [6]. 
This study aimed to detect prolonged poliovirus excretion in children later diagnosed with congenital antibody deficiency disorders.

\section{Methodology}

Eligibility criteria

A cohort of fifteen children diagnosed with primary antibody deficiency and/or specific syndromes with defects in antibody function were recruited from the Primary Immunodeficiency Disorders Clinic at Cairo University for the study from 2006 through 2009. Patients were diagnosed with antibody deficiency disorders if there was a marked decrease of immunoglobulins (at least 2 SD below the mean for age) according to the International Union of Immunological Societies (IUIS) Primary Immunodeficiency Classification Committee [9]. Informed consent from participants and approval from the research review board of our hospital were obtained prior to inclusion.

Patients who had exposure to OPV during the first year of life (before their immunodeficiency was diagnosed) and/or boosters on National Immunization Days were eligible for the study. The outcome measured was the excretion of poliovirus in stool samples. Repeat samples were taken monthly in follow-up sessions from patients who tested positive for virus excretion. Patients underwent detailed history taking and comprehensive medical examination with emphasis on the following:

- family history (consanguinity, affected siblings, similar conditions)

- presenting symptoms of Primary Immune Deficiency

- vaccination history (compulsory, boosters, any side effects)

- initiation of replacement therapy by intravenous immunoglobulins, compliance to treatment

Immunological assessment included complete blood counts; T, B and NK cell subsets by flow cytometry (using FACSE Pics, coulter) CD3, CD4, CD8, CD56, CD19; and immunoglobulins (radio immunoassay).

\section{Exclusion criteria}

Defined causes of secondary hypogammaglobulinemia such as nephrosis and chronic diarrhea, patients with other immunodeficiency disorders with intact antibody functions, and/or older antibody deficiency patients over ten years of age were all excluded from the study. Specimen collection and processing
Fecal specimens were collected at one-month intervals and patients with negative virus shedding for three successive samples were excluded. Samples were collected from patients and transferred to the laboratory under strict precautions for conservation purposes.

\section{Virus isolation and serotyping}

Specimens were treated and cultured on RD (cell line derived from human rhabdomyosarcoma) and L20B (mouse cell line expressing the gene for the human cellular receptors for poliovirus) using standard methods [10]. For positive samples, isolated viruses were characterized by intratypic differentiation (ITD) using two methods: (i)enzyme-linked immunosorbent assay (ELISA); and (ii) reverse transcription polymerase chain reaction (Rt-PCR).

\section{Nucleotide sequencing}

Purified viral RNA was amplified by RT-PCR, and the products were sequenced using an ABI Prism 310 Genetic Analyzer (Applied Biosystems, Foster City, CA, USA).

Statistical methods: The descriptive statistics were presented with mean +/- standard deviation (SD) for continuous variables and proportion for categorical variables. Student's t-test or Mann-Whitney test were used to compare continuous variables.

\section{Results}

The study included fifteen patients diagnosed with antibody deficiency disorders. There were eleven males and four females. The ages ranged between three and eight years with a mean of five years and a standard deviation of 1.66. Diagnoses included four cases of agammaglobulinemia, four cases of hypogammglobulinemia, three cases of common variable immunodeficiency disorder (CVID), three cases of ataxia telangiectasia, and a single case of combined immunodeficiency. The mean immunoglobulin G level was $142 \mathrm{mg} / \mathrm{dl}$, SD 55 (range $80-283 \mathrm{mg} / \mathrm{dl}$ ). Table 1 represents the patients' demographic and immunoglobulin profiles.

The average duration of immunoglobulin therapy prior to sample collection was equal to four months. Sampling was performed prior to the monthly transfusion visit. Each patient had received at least three compulsory doses of OPV as well as boosters, as recorded in their vaccination booklets; results varied between cases (prior to diagnosis). Family history of polio-like illness was negative in all subjects. 
Table 1.Demographic and clinical data of patients with primary immunodeficiency tested for poliovirus excretion

\begin{tabular}{|c|c|}
\hline Mean age/year (SD) & $5(1.66)$ \\
\hline Sex Male: Female & $11: 4$ \\
\hline Mean age at diagnosis/year (SD) & $4.2(1.9)$ \\
\hline Presenting symptoms & $\begin{array}{l}\text { Sino pulmonary infections } 86 \% \\
\text { Failure to thrive } 80 \% \\
\text { Fever } 53 \% \\
\text { Chronic diarrhea } 30 \% \\
\text { Neurological symptoms } 13 \% \\
\text { Joint affections } 6 \% \\
\text { Skin manifestations } 6 \%\end{array}$ \\
\hline $\begin{array}{l}\text { Family history } \\
\text { Positive consanguinity } \\
\text { Similar conditions }\end{array}$ & $\begin{array}{l}73 \% \\
53 \%\end{array}$ \\
\hline Final diagnosis & $\begin{array}{l}\text { Agammaglobulinemia }(\mathrm{n}=4) \\
\text { Hypogammglobulinemia }(\mathrm{n}=4) \\
\text { CVID }(\mathrm{n}=3) \\
\text { Ataxia telangectisia }(\mathrm{n}=3) \\
\text { Combined immunodeficiency }(\mathrm{n}=1)\end{array}$ \\
\hline $\begin{array}{l}\text { Mean immunoglobulin profiles (SD) } \\
\text { IgG } \\
\text { IgM } \\
\text { IgA } \\
\text { Mean CD3 \% Pan T cells (SD) } \\
\text { Mean CD 4\% Helper Tcells (SD) } \\
\text { Mean CD8 \% Cytotoxic Tcells (SD) } \\
\text { Mean CD19 \% B cell marker (SD) }\end{array}$ & $\begin{array}{l}142 \mathrm{mg} / \mathrm{dl},(55) \\
112 \mathrm{mg} / \mathrm{dl} \mathrm{(322)} \\
29 \mathrm{mg} / \mathrm{dl} \mathrm{(33)} \\
67.5 \%(21) \\
37.6 \%(16) \\
25.8 \%(13) \\
9.5 \%(13)\end{array}$ \\
\hline $\begin{array}{l}\text { Mean duration of immunoglobulin therapy prior to } \\
\text { sampling (SD) }\end{array}$ & 4 months $(1.1)$ \\
\hline
\end{tabular}

From 44 stool specimens, 4 obtained from two patients were positive. Serotype 2 was isolated from the first specimen obtained from the patient suffering from ataxia telangectisia (Case 3), with no virus shedding detected in the next two successive samples. Serotype 1 was isolated from three successive samples obtained from the patient suffering from common variable immunodeficiency (Case 5). The last OPV dose before the stool samples were taken was three months earlier for Case 3 and four months for Case 5.

The standard follow-up time was three months but follow-up was increased in cases of positive excretion until samples reverted to negative. Case 5 was lost to follow-up for six months and later presented with negative stool samples.
Poliovirus isolates were analyzed by ELISA and Rt-PCR. SL1 and SL2 were detected from patients 5 and 3 respectively. Nucleotide sequencing of $\mathrm{Vp} 1$ to $\mathrm{A}$ region was performed on the four isolates. Comparison with the corresponding region in the Sabin strain revealed that all were Sabin-like.

\section{Non-polio enteroviruses}

Non-polio enteroviruses were not isolated from any specimens.

\section{Discussion}

This study highlights the potential future risk of polio acquisition through prolonged excretors propagating spread of the disease in the community in an era where vaccination would be stopped because of eradication of wild virus. Patients with both primary 
[11-13] and secondary immunodeficiency disorders have been proved to excrete the virus in a prolonged manner [14-16].

In our study, SL2 was isolated from the first sample from a patient suffering from ataxia telangiectasia and no virus shedding was detected in the next two successive samples. These two samples were taken from the patient after starting immunoglobulin therapy. SL1 was isolated from the CVID patient with zero IgA levels for three successive samples taken before starting regular immunoglobulin therapy; she was lost to follow-up for six months and later presented with negative samples. The fact that this patient in particular was non-compliant to intravenous immunoglobulin therapy may suggest that excretion patterns may be different if the other patients were not receiving their replacement therapy of IVIG. The IgA deficiency is also associated with longer times of poliovirus excretion [4].

In Egypt OPV is recommended to be administered to all children below five years of age for routine immunization and NIDs. In our study, all participants received OPV before being diagnosed as immunodeficient. Two cases out of fifteen demonstrated prolonged excretion, one transiently and the other for three consecutive samples. As OPV-like isolates differ from their respective parental strains by $<1 \%$ of VP1 nucleotides, and as nucleotide substitution accumulates at an overall rate of $<1 \%$ per year at all sites and $<3 \%$ per year at synonymous sites [7], molecular analyses of excreted viruses revealed that they are Sabin-like strains and from recent infections. The last OPV dose before the stool samples were taken was three months earlier for Case 3 and four months earlier for Case 5, suggesting recent acquisition of the excreted viral strains.

Absence of non-polio enteroviruses excretion in the study could be explained by seasonality and the number of samples. Normally around 5\% isolation is achieved by the laboratory annually.

Primary immune deficiency disorders (PID) are under-recognized in developing countries because of lack of awareness and diagnostic facilities. Although considered rare disorders, their presence is anticipated at higher rates because of the high consanguinity rates and in-breeding in the North African community [17]. Incidence of predominantly antibody disorders is estimated at around $56 \%$ of PID disorders according to the European Society of Immunodeficiency Disorders Registry [18]. A study of a PID cohort of children in Egypt stated antibody deficiency disorders constituted $35 \%$ of their study population [19].
People with some variants of antibody deficiency do not develop signs or symptoms of immunodeficiency until they are young adults [20], and such patients also constitute an unrecognized threat. The studied number of patients represents a cohort following up at a tertiary referral center and is not reflective of the prevalence of antibody deficiency disorders in the community, which is recognized as a limitation to this study. Better awareness of PID should be promoted for better identification and proper assessment of the magnitude of prolonged viral excretion among those children, especially the unrecognized, untreated children who pose a threat to their communities.

Live vaccination should be deferred when there is suspicion of PID until assessment occurs, and compliance to replacement IVIG therapy is crucial to prevent complications in confirmed cases. A larger scale multicentre study is needed for the determination of the pattern of excretion of poliovirus after better recognition of PID disorders occurs.

This study demonstrates the low occurrence of prolonged excretion of vaccine polioviruses in a group of high-risk children. Alternative factors favoring persistence of poliomyelitis virus in the environment also need to be sought.

\section{References}

1. Yang C, Chen H,Jorba J Sun HC, Yang SJ, Lee HC, Huang YC, Lin TY, Chen PJ, Shimizu H, Nishimura Y, Utama A, Pallansch M, Miyamura T, Kew O, Yang JY(2005) Intratypic recombination among lineages of type 1 vaccine derived poliovirus emerging during chronic infection of an immunodeficient patient. J Virol 79: 12623-12634.

2. Sutter RW, Kew OM, Cochi SL (2003) Poliovirus Vaccine.In Plotkin SA and Orenstein WA, editors.Vaccines.4th ed. Philadelphia: W.B. Saunders Company. 651-705.

3. Bellmunt A, May G, Zell R, Pring-Akerblom P, Verhagen W, Heim A (1999) Evolution of poliovirus type I during 5.5 years of prolonged enteral replication in an immunodeficient patient. Virology 265: 178-184.

4. Kew OM, Sutter RW, de Gourville EM, Dowdle WR, Pallansch MA (2005) Vaccine-derived polioviruses and the endgame strategy for global polio eradication. Annu Rev Microbiol 59: 587-635.

5. Kew OM, Sutter RW, Nottay B, McDonough M, Prevots DR, Quick L, Pallansch M (1998) Prolonged replication of a type 1 vaccine-derived poliovirus in an immunodeficient patient. $\mathrm{J}$ Clin Microbiol 36: 2893-2899.

6. Martin J, Dunn G, Hull R, Patel V, Minor (2000) Evolution of the Sabin strain of type 3 poliovirus in an immunodeficient patient during the entire 637-day period of virus excretion. $\mathrm{J}$ Virol 74: 3001-3010.

7. Yoneyama T, Hagiwara A, Hara M, Shimojo (1982) Alteration in oligonucleotide fingerprint patterns of the viral genome in poliovirus type 2 isolated from paralytic patients. Infect Immun 37: 46-53. 
8. Halsey N, Pinto J, Espinosa-Rosales F, Faurie-Fontenla M, da Silva E, Khan A, Webster A, Minor P, Dunn G, Austrias E, Hussain H, Pallansch M, Kew M, Winkelstein J, Sutter R (2004) Search for poliovirus carriers among people with primary immune deficiency diseases in the United States, Mexico, Brazil, and the United Kingdom. Bull World Health Organ vol.82 no.1 Geneva.

9. Geha RS, Notarangelo LD, Casanova JL (2007) Primary immunodeficiency diseases: an update from the International Union of Immunological Societies Primary Immunodeficiency Diseases Classification Committee. J Allergy Clin Immunol 120: 776-794.

10. World Health Organization (2004). Polio laboratory manual, 4th edition. Geneva: World Health Organization. (WHO/EPI/GEN/97.01) Ordering code: WHO/IVB/04.10.

11. Hidalgo S (2003) Paralytic poliomyelitis caused by a vaccinederived polio virus in an antibody-deficient Argentinean child Pediatr Infect Dis J 22: 570-572.

12. Odoom JK (2008) Changes in population dynamics during long-term evolution of sabin type 1poliovirus in an immunodeficient patient J Virol 82: 9179-9190.

13. Buttinelli G, Donati V, Fiore S, Marturano J, Plebani A, Balestri P, Soresina AR, Vivarelli R, Delpeyroux F, Martin J, Fiore L (2003) Nucleotide variation in Sabin type 2 poliovirus from an immunodeficient patient with poliomyelitis J Gen Virol 84: 1215-1221.

14. Khetsuriani N (2009) Limited duration of vaccine poliovirus and other enterovirus excretion among human immunodeficiency virus infected children in Kenya, BMC Infect Dis 9: 136-139.

15. Hennessey KA (2005) Poliovirus vaccine shedding among persons with HIV in Abidjan, Cote d'Ivoire J Infect Dis 192: 2124-2128.
16. Asturias EJ (2006) Poliovirus excretion in Guatemalan adults and children with HIV infection and children with cancer. Biologicals 34: 109-12.

17. Barbouche MR, Galal N, Ben-Mustapha I, Jeddane L, Mellouli F, Ailal F, Bejaoui M, Boutros J, Marsafy A, Bousfiha AA (2011) Primary immunodeficiencies in highly consanguineous North African populations. Ann NY Acad Sci 1238: 42-52.

18. Gathmann B, Grimbacher B, Beaute J, Dudoit J, Mahlaoui N, Fischer A, Knerr V, Kindle G, ESID Working Party (2009) The European internet-based patient and research database for primary immunodeficiencies: results 2006-2008. Clinical and Experimental Immunology 157: 3-11.

19. Reda S, Afifi H, Amine M (2009) Primary Immunodeficiency Diseases in Egyptian Children: A Single-Center Study J Clin Immunol 29: 343-351.

20. Cunningham-Rundles C and Bodian C (1999) Common variable immunodeficiency: clinical and immunological features of 248 patients. Clinical Immunology 92: 34-48.

\section{Corresponding author}

Nermeen M Galal,MD-FRCPCH

Associate Professor of Paediatrics

Cairo University

1 A Road 233 Degla Maadi

Cairo, Egypt

Email: Nermeengalal@gmail.com

Telephone: +201001643197

Conflict of interests: No conflict of interests is declared. 